\title{
ANÁLISE DA PRODUÇÃO DE ARTEFATOS DE MADEIRA PELO MÉTODO DE CUSTEIO BASEADO EM ATIVIDADES
}

\author{
Álefe Lopes Viana ${ }^{1 *}$, Fernando Cardoso Lucas Filho ${ }^{2}$, Nara Medianeira Stefano ${ }^{3}$ \\ 1* Instituto Federal de Educação, Ciência e Tecnologia do Amazonas, Lábrea, Amazonas, Brasil - alefe.viana@ifam.edu.br \\ ${ }^{2}$ Universidade Federal do Amazonas, Departamento de Ciências Florestais, Manaus, Amazonas, Brasil - fecarlu@ufam.edu.br \\ ${ }^{3}$ Universidade Federal de Santa Catarina, Florianópolis, Santa Catarina, Brasil- stefano.nara @ gmail.com
}

Recebido para publicação: 22/07/2015 - Aceito para publicação: 27/06/2016

\begin{abstract}
Resumo
A baixa atratividade econômica das atividades florestais tem sido uma das várias causas do desmatamento na Amazônia. A produção de móveis e artefatos industrializados de madeira seria uma alternativa, mas devido à ausência de competitividade dos produtos produzidos na região essa atividade ainda não se comprovou esse pressuposto. Uma das formas de obter melhorias é conhecer os custos relacionados aos processos, visando identificar causas e propor soluções de engenharia para reduzir essa ineficiência. Baseado nesta premissa, a pesquisa usou o método de custeio baseado em atividades (ABC) para analisar os fatores envolvidos no processo de produção de um artefato produzido com madeira da Amazônia. Foi usada a técnica de cronoanálise que permitiu a elaboração do mapa do fluxo do processo. As atividades que mais agregaram valor foram lixamento, colagem/montagem e aplicação de selador. Os resultados mostram que as atividades que menos agregaram valor, caracterizadas por seu alto tempo de setup e medições, foram o esquadrejamento, furação e confecção de espigas. Custos com materiais foram superiores à mão de obra. A participação dos custos com a madeira em relação ao custo final do produto não ultrapassou $14 \%$.

Palavras-chave: Produtos de madeira; Activity Based Costing (ABC); custos de produção; Amazônia.
\end{abstract}

\begin{abstract}
Analysis of production of artifacts of costing method for wood-based activities. Low economic attractiveness of forestry activities has been one of several causes of deforestation in the Amazon. The furniture production and industrial wood artifacts would be an alternative, but due to lack of competitiveness of the products produced in the region this activity is not yet proved this assumption. One way to achieve improvements is to know the costs related to the processes, to identify causes and propose engineering solutions to reduce this inefficiency. Based on this premise, the research used the costing method based on activities (ABC) to analyze the factors involved in the production process of an artifact produced with wood from the Amazon. It was used to chronoanalysis technique that allowed the development of the process flow map. The activities that add value have been sanding, bonding / assembly and application of sealer. The results show that the activities less added value, characterized by its high time to setup and measurements were squaring, drilling and production of corn. Material costs were higher than the labor. The share of costs with wood from the end cost of the product did not exceed $14 \%$.
\end{abstract}

Keywords: Wood products; Activity Based Costing (ABC); production costs; Amazon.

\section{INTRODUÇÃO}

A abertura da economia, a sua estabilização e a presença cada vez mais marcante de negócios com abrangência global, exigiram a substituição do antigo modelo de gerenciamento empresarial, baseado na produtividade, por um novo modelo baseado na competitividade. Assim, o desenvolvimento de vantagem competitiva (LI et al., 2012; SOLODUCHO-PELC, 2014; HERRERA, 2015) depende da capacidade da organização de se diferenciar dos concorrentes por meio da busca do equilíbrio entre os objetivos de prazo, qualidade, inovação e, principalmente, de custo.

Concorrência, inovação e globalização são termos que por algumas décadas têm estado presentes em quase todos os aspectos da atividade social ou econômica. Em particular, o crescente interesse em questões relacionadas com a competitividade tem contribuído para o desenvolvimento de teorias e estudos no âmbito da análise da concorrência e as estratégias de competição.

$\mathrm{Na}$ indústria de base florestal (NAGUBADI; ZHANG, 2011; SIERRA-PÉREZ; BOSCHMONARTRIVES; GABARRELL, 2015) não é diferente. A gestão do negócio passou a tomar um papel importante para a sustentabilidade das atividades. A melhoria da competitividade nesse segmento depende do desempenho dos processos de fabricação e esses dependem da identificação dos fatores tecnológicos e organizacionais 
responsáveis pela eficiência produtiva nas indústrias de móveis.

Muitas forças diferentes estão moldando a evolução da indústria de base florestal (NÄYHÄ; PESONEN, 2014), e estes têm consequências para a sua continuidade. Algumas das forças mais proeminentes são amplamente negativas (por exemplo, a estrutura da indústria e da maturidade de alguns mercados) e só podem ser abordados por mudanças dentro da própria indústria.

Assim vários fatores (BONDUELLE, 1997; NAUMANN, 1998; COUTINHO, 1999; TOMASELLI, 2000; LUCAS FILHO, 2004) contribuem para os problemas de competitividade no segmento madeira-móveis, por exemplo, a má gestão dos recursos produtivos, ausência de um design próprio e mais atraente, organização industrial pouco desenvolvida, cultura industrial atrasada, ausência de certificação da madeira, ausência de estratégias comerciais e de marketing competitivas e tecnologia de fabricação ineficiente, gerando maiores custos e altos índices de perdas na produção, além do baixo nível da qualidade final dos produtos.

Todos estes fatores causam a baixa atratividade econômica da atividade florestal, iniciada ainda no manejo florestal (LEHTONEN; TYKKYLÄINEN, 2014), o que chega a abrir precedentes para o aumento do desmatamento (ARIMA et al., 2014; PANDAY et al., 2015), tendo como exemplo alguns fatores como a baixa produtividade da floresta e lacunas entre os elos da cadeia produtiva, onde uma das soluções apontadas seria o desenvolvimento de produtos com alto valor agregado.

Dessa forma, uma das formas de melhoria é conhecer os custos relacionados aos processos de produção (NG; THIRUCHELVAM, 2012), visando soluções palpáveis. Para isso, os velhos conceitos da contabilidade de custos devem ser substituídos por novos conceitos. Os rateios simplistas pregados pela contabilidade de custos tradicional não mais atendem aos reclamos dessa nova era, sob o risco de deixarem as empresas com custos distorcidos.

O Activity-Based Costing (ABC) (NAKAGAWA, 1994; NASSAR et al., 2013; LELKES, 2014; CANNAVACCIUOLO et al., 2015), Custeio Baseado em Atividades, é um método de custeio que foi desenvolvido com o objetivo de aprimorar a alocação dos custos indiretos que eram direcionados pela arbitrariedade do rateio utilizado nos sistemas tradicionais, permitindo um controle mais efetivo dos gastos. $\mathrm{O}$ $\mathrm{ABC}$ contempla todo o processo produtivo, buscando identificar ociosidades e atividades que não agregam valor, constituindo-se, desta forma, em uma ferramenta para tomada de decisões.

De maneira geral é um método econômico de atividades relacionadas com a produção de uma organização. A relação causal entre os produtos e os clientes que consomem recursos é determinada pelo custo de rastreamento com base em um fator (driver do custo) que causa ou se correlaciona altamente com o uso de um produto ou cliente da de recursos de uma atividade.

Diante desse cenário, este trabalho busca contribuir com soluções para o problema exposto fornecendo suporte às tomadas de decisões gerenciais de custeio da produção de artefatos de madeira, com base no mapeamento do processo produtivo, haja vista que no setor industrial madeireiro vários estudos buscam identificar as atividades que agregam e, principalmente, as que não agregam valor aos produtos levando a uma maior eficiência na gestão dos processos produtivos.

Partindo desse pressuposto, este estudo tem o objetivo de utilizar o sistema de custos baseado em atividades $(\mathrm{ABC})$ como ferramenta de planejamento e apoio às decisões na produção de artefatos de madeira.

\section{MATERIAL E MÉTODOS}

Para a coleta de dados, foi realizado um estudo de caso no Laboratório-marcenaria da Universidade Federal do Amazonas, o qual dá suporte aos trabalhos acadêmicos de graduação e pós-graduação. Foram produzidas poltronas com madeira (Figura 1) da espécie Cariniana micrantha Ducke (SCHLESER et al., 2015), conhecida popularmente como Tauari-vermelho - árvore da família das Lecythidaceae, sendo escolhida devido à grande abundância nas madeireiras da Cidade de Manaus bem como também por ser uma madeira não tão apreciada para a produção de móveis e artefatos, sendo mais utilizada na construção civil, como escora para lajes, produtos de baixo valor agregado.

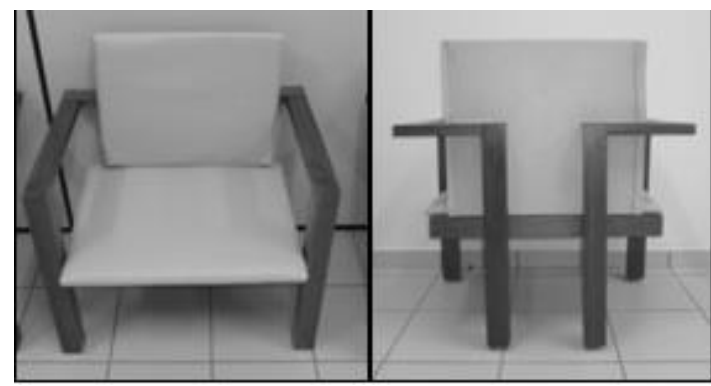

Figura 1. Poltrona produzida com madeira de Tauari-vermelho.

Figure 1. Armchair produced with wood Red-Tauari. 
Nesta pesquisa, estão apresentados os processos produtivos e as suas respectivas atividades, desempenhadas na produção do móvel. Foram identificadas as atividades, atribuindo os custos às tarefas e apontando os recursos que foram consumidos, com base no tempo de execução de cada atividade inerente ao processo produtivo.

O custo de produção foi obtido pelo método do Custeio Baseado em Atividades ( $\mathrm{ABC}$ ), onde foram identificados e estruturados os recursos utilizados. A fundamentação metodológica seguiu conforme descrita nos modelos de aplicação desenvolvidos por Nakagawa (1994), Kaplan e Cooper (1998) e Ching (1997), onde, qualquer metodologia de implantação do ABC (SILVA, 2000) deve ser adaptada às necessidades requeridas e aos objetivos de sua implantação.

Após identificadas e agrupadas as atividades, foi realizado o levantamento dos custos de produção, buscando dados de descrição das tarefas e insumos utilizados. Desta forma, para calcular o Custo Total de Produção ou custo fabril (CP), foram somados os custos de matéria-prima, mão de obra e despesas (energia elétrica e depreciação).

\section{RESULTADOS E DISCUSSÃO}

Um dos problemas limitantes para a pesquisa foi a ausência de literatura para comparação dos resultados da fabricação de produtos de madeira na região. Muitas das pesquisas com ABC desenvolvidas no setor florestal são apenas aplicadas no setor da silvicultura e manejo florestal.

Para alocação dos custos, foi necessário construir a tabela de atividades, com seus respectivos direcionadores, em ordem de produção, como mostrado na tabela 1.

Tabela 1. Direcionadores de atividades.

Table 1. Drivers activity.

\begin{tabular}{|c|c|c|c|}
\hline Ordem & Atividade & Descrição/Objetivo da atividade & Direcionador \\
\hline 1 & Corte / Desdobro & Desdobro secundário para transformação de peças menores & Tempo de corte \\
\hline 2 & Aplainamento & $\begin{array}{c}\text { Eliminar rugosidade da superfície das peças bem como } \\
\text { eliminar empenamentos }\end{array}$ & $\begin{array}{l}\text { Tempo de } \\
\text { aplainamento }\end{array}$ \\
\hline 3 & Desengrosso & Controlar a bitola das peças & Tempo de desengrosso \\
\hline 4 & Esquadrejamento & Controle do comprimento das peças & Tempo de corte \\
\hline 5 & Furação & $\begin{array}{c}\text { Operação de desbaste feito com brocas helicoidais para } \\
\text { encaixe das espigas e parafusos }\end{array}$ & Tempo de furação \\
\hline 6 & Produção de espigas & Produzir encaixes feitos pela serra circular & Tempo de corte \\
\hline 7 & $\begin{array}{l}\text { Colagem / } \\
\text { Montagem }\end{array}$ & Unir/Agrupar as várias peças que compõem o produto & Tempo de execução \\
\hline 8 & Lixamento & $\begin{array}{c}\text { Operação de acabamento que confere melhor aderência ao } \\
\text { selador }\end{array}$ & Tempo de lixamento \\
\hline 9 & Aplicação de selador & Acabamento final usado para conservação do produto & Tempo de aplicação \\
\hline
\end{tabular}

\section{Mapeamento do processo de produção}

Com o mapeamento do processo definido, foi possível coletar os tempos respectivos de produção de cada atividade. Em relação aos tempos de execução, as atividades que consumiram maior tempo foram lixamento, colagem/montagem e aplicação de selador, ambas com 93,312 min, 75,054 min e 53,130 min, respectivamente, tempo este caracterizado como transformador da matéria-prima. A figura 2 apresenta os tempos de execução de cada atividade:

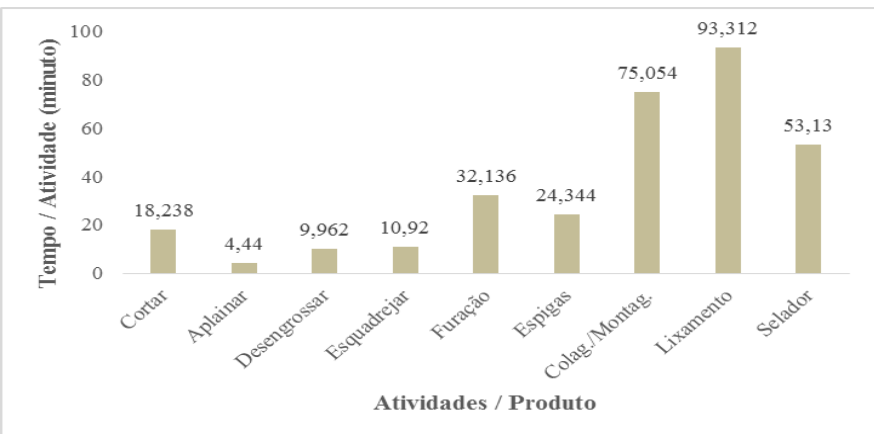

Figura 2. Tempo total das atividades do processo (incluindo setup e medição).

Figure 2. Total time of process activities (including setup and measurement).

FLORESTA, Curitiba, PR, v. 46, n. 4, p. 447 - 454, out. / dez. 2016

Viana, A. L. et al.

ISSN eletrônico 1982-4688

DOI: $10.5380 /$ rf.v46i3.42220 
As três juntas somam quase $69 \%$ do tempo empregado para produção de uma poltrona, com destaque para o lixamento, atividade que mais consumiu tempo, como mostrado na tabela 2. As atividades com menor tempo de execução foram aplainamento, desengrosso e esquadrejamento, ambos 4,440 min., 9,962 min. e 10,920 min., respectivamente. É importante ressaltar que os dados utilizados para o gráfico apresentado na figura 2 incluem dados de operação, medição/marcação e tempo de setup, ambos, juntos.

Tabela 2. Proporção do tempo médio de execução utilizado em cada atividade.

Table 2. Proportion of average execution time spent on each activity.

\begin{tabular}{lcc}
\hline Atividades & Tempo (min.) & Proporção (\%) \\
\hline Cortar & 18,238 & 5,69 \\
Aplainar & 4,440 & 1,39 \\
Desengrossar & 9,962 & 3,11 \\
Esquadrejar & 10,920 & 3,41 \\
Furação & 32,136 & 10,02 \\
Espigas & 24,344 & 7,60 \\
Colag./Mont. & 75,054 & 23,41 \\
Lixamento & 92,312 & 28,80 \\
Selador & 53,130 & 16,57 \\
\hline Total & $\mathbf{3 2 0 , 5 3 6}$ & $\mathbf{1 0 0}$ \\
\hline
\end{tabular}

A atividade de lixamento se destacou devido à quantidade de operações em uma sequência de lixamento, sendo lixa grão 50,80,100,120,150, 180 e 220. A segunda maior atividade que consumiu tempo foi a colagem/montagem da poltrona, com cerca de $23 \%$ do tempo total consumido. Cabe fazer alusão a essa atividade pelo fato de que tal processo foi dividido em duas partes: a montagem estrutural da poltrona (feita na marcenaria) e a montagem de assento e encosto (feita na tapeçaria). Cerca de $38 \%$ da operação foi feita na tapeçaria, onde primeiro foram montados assentos e encostos (com painel compensado revestido de espuma e tecido corvin).

Com relação aos tempos de medição/marcação de peças, a atividade que mais consumiu tempo foi a atividade de furação, com 10,23 minutos/produto. A precisão requerida na operação de furação para encaixe das espigas desprendeu muito tempo por não existir um gabarito que facilitasse o processo. Além do mais, várias peças que compõem a estrutura foram furadas (braços, pernas e assentos e encostos) e para cada operação houve a necessidade de marcar a posição dos furos para poder ser equivalente ao tamanho da espiga que o receberia.

Cabe ressaltar que somente as atividades de corte, furação, espigas e colagem/montagem tiveram tempos de medição, devido à necessidade de precisão de execução, uma vez que se a operação não existisse, as demais atividades sequenciais não sairiam conforme o projeto do produto. Na colagem/Montagem a medição que houve ocorreu na tapeçaria, para divisão dos tecidos e espuma, que no mercado são comercializados por metro.

Quanto aos tempos de setup, as atividades com maior tempo em execução foram furação, lixamento e esquadrejamento com 11,9 $\mathrm{min}, 8,8 \mathrm{~min}$ e $6,7 \mathrm{~min}$, respectivamente. A tabela 3 apresenta os tempos de setup para todas as atividades.

Tabela 3. Tempo de setup médio com intervalo de confiança a $90 \%$.

Table 3. Average Time setup with a confidence interval of $90 \%$.

\begin{tabular}{lcc}
\hline Atividades & Tempo (min.) & Proporção (\%) \\
\hline 1. Cortar & $3,432 \pm 0,051$ & 7,59 \\
2. Aplainar & $0,400 \pm 0,010$ & 0,88 \\
3. Desengrossar & $3,116 \pm 0,015$ & 6,89 \\
4. Esquadrejar & $6,702 \pm 0,083$ & 14,82 \\
5. Furação & $11,900 \pm 0,743$ & 26,31 \\
6. Espigas & $4,552 \pm 0,123$ & 10,06 \\
7. Colag./montg. & $4,400 \pm 0,057$ & 9,73 \\
8. Lixamento & $8,886 \pm 0,215$ & 19,65 \\
9. Selador & $1,840 \pm 0,030$ & 4,07 \\
\hline Total & $\mathbf{4 5 , 2 2 8}$ & $\mathbf{1 0 0}$ \\
\hline
\end{tabular}

A Furação consumiu maior tempo de setup devido ao fato de várias peças da poltrona receberam furos para encaixe das espigas, com encaixes diferentes.

\section{Determinação dos custos}

Os dados foram compilados na tabela 4, contendo custos com mão de obra, energia, depreciação e materiais. 
Tabela 4. Custo médio por atividade do processo produtivo da poltrona.

Table 4. Average cost per activity of the productive process of the chair.

\begin{tabular}{lccccccc}
\hline Item & \multirow{2}{*}{ Atividade } & $\begin{array}{c}\text { M.O. } \\
\mathbf{( R \$ )}\end{array}$ & $\begin{array}{c}\text { Energia } \\
(\mathbf{R} \mathbf{)}\end{array}$ & $\begin{array}{c}\text { Depreciação } \\
(\mathbf{R} \mathbf{)}\end{array}$ & $\begin{array}{c}\text { Materiais } \\
(\mathbf{R} \mathbf{)}\end{array}$ & $\begin{array}{c}\text { Total / Atividade } \\
(\mathbf{R} \mathbf{)})\end{array}$ & $\begin{array}{c}\text { \% de } \\
\text { participação }\end{array}$ \\
\hline 1 & Cortar & 6,501 & 0,109 & 0,156 & 57,655 & 64,421 & 26,63 \\
2 & Aplainar & 0,671 & 0,060 & 0,096 & 0,000 & 0,827 & 0,34 \\
3 & Desengrossar & 2,029 & 0,093 & 0,149 & 0,270 & 2,541 & 1,05 \\
4 & Esquadrejar & 1,525 & 0,057 & 0,092 & 0,500 & 2,174 & 0,90 \\
5 & Furação & 4,488 & 0,108 & 0,163 & 0,269 & 5,028 & 2,08 \\
6 & Espigas & 3,400 & 0,122 & 0,195 & 1,000 & 4,717 & 1,95 \\
7 & Colag./mont. & 27,711 & 0,050 & 0,004 & 101,037 & 128,802 & 53,23 \\
8 & Lixamento & 12,893 & 0,177 & 0,045 & 9,729 & 22,844 & 9,44 \\
9 & Selador & 7,420 & 0,000 & 0,000 & 3,182 & 10,602 & 4,38 \\
\hline & TOTAL & $\mathbf{6 6 , 6 3 7}$ & $\mathbf{0 , 7 7 6}$ & $\mathbf{0 , 9 0 0}$ & $\mathbf{1 7 3 , 6 4 2}$ & $\mathbf{2 4 1 , 9 5 6}$ & $\mathbf{1 0 0}$ \\
\hline
\end{tabular}

Os resultados mostram que dentre os direcionadores de custos do processo produtivo, os que mais consumiram recursos foram matéria-prima e mão de obra, $\mathrm{R} \$ 173,642$ e $\mathrm{R} \$ 66,637$, respectivamente. Custos com energia e depreciação foram baixos devido ao pouco tempo de uso das máquinas. As atividades de aplicação de selador e colagem/montagem (marcenaria) não computaram gastos com energia e depreciação, pois foram usados apenas materiais de consumo e suas operações foram executadas pelo marceneiro, ou seja, apenas consumiu recursos para mão de obra.

Para o direcionador mão de obra, a atividade que mais consumiu recursos foi a colagem/montagem, seguido de lixamento e aplicação de selador, onde está atrelado mais de 70\% do custo médio da M.O., 41,58\%, $19,34 \%$ e $11,13 \%$, respectivamente.

A participação da mão de obra do processo de montagem/colagem foi alta devido ao fato de esta ser subdividida em duas partes: marcenaria e tapeçaria. Dos poucos mais de R $\$ 27$ consumidos pela atividade, cerca de R \$ 20 foi consumido pela mão de obra das operações ocorridas na tapeçaria.

Cabe aqui a análise de duas atividades - esquadrejamento e furação: ambas possuíram tempo de setup e medição superior ao tempo propriamente dito de execução da atividade. Do total de 10,92 minutos do tempo consumido no esquadrejamento, mais de $61 \%$ é destinado a setup. Isso é explicado também pelo fato de que para cada comprimento de peça da poltrona houve uma adequação da máquina para que o corte fosse feito na medida certa. A poltrona possui 6 componentes de diferentes medidas e isso aumenta o tempo de parada para setup e adaptação da máquina.

A furação também apresenta valores de tempo para setup e medição muito discrepantes. Cerca de $68 \%$ é consumido por setup's e medições. Mais de $31 \%$ da atividade é consumida pela operação de medição das peças para furação, necessária até então por não ter uma respigadeira que realiza o trabalho facilmente, fazendo com que a agregação de valor ao produto seja muito baixa (Figura 3).

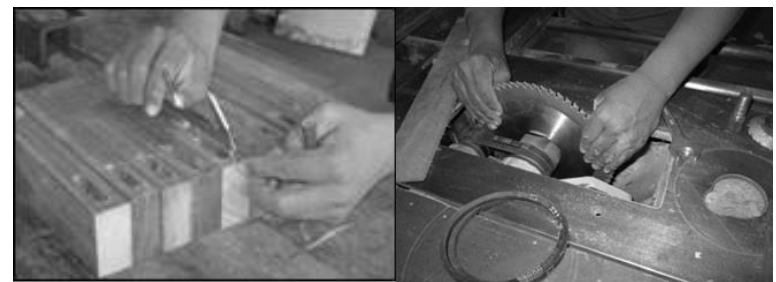

Figura 3. Medição dos furos para encaixe das espigas com uso de paquímetro (à esq.) e setup da serra circular (à dir.). Figure 3. Measurement of holes to plug the ears with a caliper (left.) And set the circular saw (right.).

Quanto ao direcionador energia elétrica, o consumo médio foi baixo, pois as atividades por unidade produzida não excederam o tempo máximo de pouco mais de 28 minutos ininterruptos, executado na atividade de lixamento, operado pela lixadeira orbital.

$\mathrm{Na}$ colagem/montagem (tapeçaria) houve gasto de energia devido ao fato de que as espumas dos encostos foram montados por meio de costura (máquina elétrica de costura). O direcionador depreciação, as atividades que mais depreciaram máquinas foram as de produção da espiga, furação e corte.

O processo de lixamento apresentou baixo valor de depreciação devido ao preço de aquisição da máquina ser muito baixo em relação às demais e consumir pouco tempo de trabalho (hora de trabalho em relação à vida útil). A atenção no processo da atividade do lixamento deve ser concentrada para redução do valor de mão de obra, devido ao tempo consumido. 


\section{Mensuração dos custos de matéria-prima}

$\mathrm{Na}$ tabela 5 são mostrados todos os materiais com suas devidas quantidades e unidades que foram consumidos no processo de produção da poltrona:

Tabela 5. Custos médios com matéria-prima para o processo de produção da poltrona.

Table 5. Average costs with raw material for the chair production process.

\begin{tabular}{llllcrc}
\hline $\mathbf{N}$ & Item & Atividade & Qtd. & Custo (RS) & Total R\$) & Participação (\%) \\
\hline $\mathbf{1}$ & Disco de serra vídea (Unid.) & Corte & 0,1 & 171,85 & 17,185 & 9,90 \\
$\mathbf{2}$ & Madeira $\left(\mathrm{m}^{3}\right)$ & Corte & 0,0221 & 1094 & 24,177 & 13,92 \\
$\mathbf{3}$ & Painel Compensado $\left(\mathrm{m}^{2}\right)$ & Corte & 0,605 & 26,93 & 16,293 & 9,38 \\
$\mathbf{4}$ & Spray óleo multiuso (ml) & Desengrosso & 10 & 0,027 & 0,270 & 0,16 \\
$\mathbf{5}$ & Esquadro (Unid.) & Esquadrejar & 0,1 & 5 & 0,500 & 0,29 \\
$\mathbf{6}$ & Broca aço 3,5 mm (Unid.) & Furação & 0,1 & 2,69 & 0,269 & 0,15 \\
$\mathbf{7}$ & Broca aço 10 mm (Unid.) & Furação & 0,1 & 10 & 1,000 & 0,58 \\
$\mathbf{8}$ & Lixas (m $\left.{ }^{2}\right)$ & Lixamento & 7,13 & 25,38 & 7,488 & 4,29 \\
$\mathbf{9}$ & Óculos de proteção (Unid.) & Lixamento & 0,1 & 5 & 0,500 & 0,29 \\
$\mathbf{1 0}$ & Máscara (Unid.) & Lixamento & 1 & 1,74 & 1,740 & 1,00 \\
$\mathbf{1 1}$ & Selador (L) & Aplicação de selador & 0,09 & 10,93 & 0,984 & 0,57 \\
$\mathbf{1 2}$ & Algodão (g) & Aplicação de selador & 0,04 & 27,8 & 1,112 & 0,64 \\
$\mathbf{1 3}$ & Estopa (g) & Aplicação de selador & 0,04 & 10 & 0,400 & 0,23 \\
$\mathbf{1 4}$ & Thinner (L) & Aplicação de selador & 0,09 & 7,63 & 0,687 & 0,40 \\
$\mathbf{1 5}$ & Cola Branca Extra-forte (Kg) & Colagem/Montagem & 0,075 & 15,63 & 1,172 & 0,69 \\
$\mathbf{1 6}$ & Parafuso (Unid.) & Colagem/Montagem & 28 & 0,08 & 2,240 & 1,29 \\
$\mathbf{1 7}$ & Sapata niquelada 30 mm (Unid.) & Colagem/Montagem & 4 & 2,43 & 9,720 & 5,60 \\
$\mathbf{1 8}$ & Cola de contato (Kg) & Colagem/Montagem & 0,025 & 25,33 & 0,633 & 0,36 \\
$\mathbf{1 9}$ & Espuma D-28 3cm (m) & Colagem/Montagem & 0,605 & 45 & 27,225 & 15,68 \\
$\mathbf{2 0}$ & Tecido Corvin (m) & Colagem/Montagem & 1,5 & 25 & 37,500 & 21,60 \\
$\mathbf{2 1}$ & Zíper (Unid.) & Colagem/Montagem & 1 & 0,5 & 0,500 & 0,29 \\
$\mathbf{2 2}$ & Tecido TNT (m) & Colagem/Montagem & 0,3 & 1,4 & 0,420 & 0,24 \\
$\mathbf{2 3}$ & Grampo marceneiro (sargento) & Colagem/Montagem & 0,2 & 108,13 & 21,626 & 12,45 \\
\hline & TOTAL & & & & $\mathbf{1 7 3 , 6 4 2}$ & $\mathbf{1 0 0}$ \\
\hline & & & & & \\
\hline
\end{tabular}

A matéria-prima que obteve maior consumo para produção da mesa foi o corvin (usado no revestimento do assento), a espuma (encosto/assento) e a madeira (estrutura).

Mais de $37 \%$ do custo dos materiais foram consumidos pelo corvin e espuma, justificado pelo valor de venda no mercado. A madeira ocupa somente a terceira posição, mesmo sendo o principal componente da estrutura (com 13,9\% dos materiais).

As atividades com maiores custos foram colagem/montagem, corte e lixamento, com R\$101,04, $\mathrm{R} \$ 57,66$ e $\mathrm{R} \$ 9,73$, respectivamente. Colagem/Montagem teve alto custo devido à espuma e o tecido que foram usados no acabamento do assento e encosto da poltrona, sobressaindo com mais de $58 \%$ do custo com materiais. Aplainamento não consumiu materiais de consumo devido à sua operação ser exclusivamente em máquina; desengrosso, esquadrejamento e furação também são atividades que usam máquinas.

\section{CONCLUSÃO}

Os resultados permitem concluir que:

- Baseado na competitividade e na acirrada concorrência, a aplicação do método de custeio ABC é de fundamental importância pois permite saber o consumo efetivo dos recursos por grupo de operações e o tempo médio de execução.

- O custeio ABC forneceu informações relevantes no processo de tomada de decisão, pois permitiu a visualização dos pontos problemáticos no fluxo das atividades que concentram esforços que não são utilizados de forma eficiente.

- As atividades que mais agregaram valor foram lixamento, colagem/montagem e aplicação de selador, onde mesmo assim ainda torna-se necessário reduzir tais custos de transformação. As atividades consideradas como gargalos foram esquadrejamento, furação e confecção de espigas.

- As atividades com maior custo médio de produção foram colagem/montagem e corte.

- Custos com matéria-prima foram superiores aos custos de mão de obra. 
- Recomenda-se, portanto, o desenvolvimento de um estudo contínuo para a melhoria dos processos, conhecido como kaizen, pois através deste é possível reavaliar e verificar o nível de importância das atividades, desenvolver novos processos que possam dar velocidade ao fluxo de produção, diminuir ou eliminar tempo de setup e medição e reduzir o custo de transformação oferecendo suporte ao gerenciamento dos processos.

\section{REFERÊNCIAS}

ARIMA, E. Y.; BARRETO, P.; ARAÚJO, E.; SOARES-FILHO, B. Public policies can reduce tropical deforestation: Lessons and challenges from Brazil. Land Use Policy, v. 41, p. 465-473, 2014.

BONDUELLE, G. M. Avaliação e análise dos custos da má qualidade na indústria de painéis de fibras. Tese (Doutorado em Engenharia de Produção) - Universidade Federal de Santa Catarina, Florianópolis, 1997.

CANNAVACCIUOLO, L.; ILlARIO, M.; IPPOLITO, A.; PONSIGLIONE, C. An activity-based costing approach for detecting inefficiencies of healthcare processes. Business Process Management Journal, v. $21 \mathrm{n}$. 1, p. 55-79, 2015.

CHING, H. Y. Gestão baseada em custeio por atividades. São Paulo: Atlas, 1997.

COUTINHO, L. Design como Fator de Competitividade na Indústria Moveleira. NEIT/ UNICAMP. RELATÓRIO SETORIAL SEBRAE - FINEP- ABIMOVEL, São Paulo, 1999.

HERRERA, M. E. B. Creating competitive advantage by institutionalizing corporate social innovation. Journal of Business Research, v. 68, n. 7, p. 1468-1474, 2015.

KAPLAN, R. S.; COOPER, R. Custo \& Desempenho: administre seus custos para ser mais competitivo. $2^{\mathrm{a}}$. ed. São Paulo: Futura, 1998.

LEHTONEN, O.; TYKKYLÄINEN, M. Delphi path simulator for unveiling development opportunities in the forest industries by contrasting forest management practices: the case of North Karelia. Technological Forecasting and Social Change, v. 84, p. 171-185, 2014.

LI, W.; HUMPHREYS, P. K.; YEUNG, A. C. L.; CHENG, T. C. E. The impact of supplier development on buyer competitive advantage: a path analytic model. International Journal of Production Economics, v. 135, n. 1, p. 353-366, 2012.

LUCAS FILHO, F. C. Análise da Usinagem da madeira visando a melhoria de processos em indústria de móveis. 176 f. Tese (Doutorado em Engenharia de produção) - Universidade Federal de Santa Catarina, Florianópolis, 2004.

NAGUBADI, R. V.; ZHANG, D. Bilateral foreign direct investment in forest industry between the U.S. and Canada. Forest Policy and Economics, v. 13, n. 5, p. 338-344, 2011.

NAKAGAWA, M. ABC: custeio baseado em atividades. São Paulo: Atlas, 1994.

NASSAR, M.; AL-KHADASH, H. A.; SANGSTER, A.; MAH'D, O. Factors that catalyse, facilitate and motivate the decision to implement activity-based costing in Jordanian industrial companies. Journal of Applied Accounting Research, v. 14 n. 1, p. 18-36, 2013.

NAUMANN, L. Tecnologia como fator de competitividade na indústria madeireira. Wood Magazine, v. 5, n. 3, p. 19-23, 1998.

NÄYHÄ, A.; PESONEN H.-L. Strategic change in the forest industry towards the biorefining business. Technological Forecasting and Social Change, v. 81, p. 259-271, 2014.

NG, B.-K.; THIRUCHELVAM, K. The dynamics of innovation in Malaysia's wooden furniture industry: Innovation actors and linkages. Forest Policy and Economics, v. 14, n. 1, p. 107-118, 2012.

PANDAY, P. K.; COE, M. T.; MACEDO, M. N.; LEFEBVRE, P.; CASTANHO, A. D. A. Deforestation offsets water balance changes due to climate variability in the Xingu River in eastern Amazonia. Journal of Hydrology, v. 523, p. 822-829, 2015.

SCHLESER, G. H.; ANHUF, D.; HELLE, G.; VOS, H. A remarkable relationship of the stable carbon isotopic compositions of wood and cellulose in tree-rings of the tropical species Cariniana micrantha (Ducke) from Brazil. Chemical Geology, v. 401, p. 59-66, 2015. 
SIERRA-PÉREZ, J.; BOSCHMONART-RIVES, J. GABARRELL, X. Production and trade analysis in the Iberian cork sector: economic characterization of a forest industry. Resources, Conservation and Recycling, v. 98, p. 55-66, 2015.

SILVA, E. R. S. O uso do ABC no gerenciamento de custos: pesquisa-ação em uma agência bancária. 167 f. Dissertação (Mestrado em Engenharia de Produção) - Universidade Federal de Santa Catarina, Florianópolis, 2000.

SOŁODUCHO-PELC, L. Competitive advantage: the courage in formulating objectives and expansiveness of a strategy. Procedia - Social and Behavioral Sciences, v. 150, p. 271-280, 2014.

TOMASELLI, I. As perspectivas para o setor florestal Brasileiro. Revista Referência, Curitiba, v. 2 n. 3, p. 28, 2000. 